\title{
CAR-T cells with co-expressed cytokines and chemokines better overcome inhibitory microenvironment of solid
}

\section{tumors}

\author{
Vita Golubovskaya ${ }^{1,2 *}$ \\ ${ }^{1}$ Probam Biotechnologies, 2600 Hilltop Drive, Richmond, CA, USA \\ ${ }^{2}$ Oklahoma Health Sciences Center, Oklahoma City, OK, USA
}

Comment on "IL-7 and CCL19 expression in CAR-T cells improves immune cell infiltration and CAR-T cell survival in the tumor", by Keishi Adachi, et al. Nature Biotechnology, E-pub March 5, 2018.

CAR (Chimeric antigen receptor)- $\mathrm{T}$ cells recently demonstrated remarkable advances in oncology in treatment of patients with hematological cancers [1-3]. The application of CAR-T cells to solid cancers meets several challenges [4] such as inhibitory tumor microenvironment, exhaustion of CAR-T cells, checkpoint inhibitors, CAR-T cell delivery and other.

The Letter report to Nature Biotechnology by Keishi Adachi, et al. March 5, 2018 describes an elegant approach to combine CD20-CAR-T cells that co-express IL-7 and CCL19, called $(7 \times 19$ CAR-T cells) [4] (Figure 1). Both IL-7 and CCL19 are critical factors to support survival of T cells in lymph nodes and lymphoid tissues. In solid tumor xenograft mice mode, these CD20 CAR-T cells had 100\% survival compared with lower survival of standard or conventional CAR-T cells. The anti-tumor effect of 7x19 CAR-T cells was the same when CD28 or 41-BB co-stimulatory domains were designed inside CAR construct [1]. In addition, these CAR-T cells expressed significantly lower levels of LAG-3, TIM-3, PD-1 and other checkpoint inhibitors compared with standard CAR-T cells which demonstrates their less exhausted phenotype. In addition, 7x19 CAR-T cells had increased migratory and increased memory functions that are key functions for increased efficacy of CAR-T cells.

One of the steps of improving CAR-T therapy was proposed by using different cytokines during CAR-T cell expansion $[5,6]$. We proposed model of increasing CAR-T cell therapy approaches (Figure 2) [6].

We tested our CAR-T cell expansion with different cytokines and detected that combination of IL-7 with other cytokines or factors increase percent of central memory cells. We demonstrate increased level of central memory cells when we expanded CD19-Flag-CAR-T cells (Figure 3). To co-express different growth factors or cytokines in CAR might be even more effective approach as demonstrated in the Nature Biotechnology report, 2018.

In conclusion the report by Keishi Adachi, et al. Nature Biotecnology, March 5, 2018 demonstrates that including different immune-regulatory factors inside CAR structure can affect not only CAR effector functions but also regulate memory functions and influence tumor microenvironment. This will lead to design of next

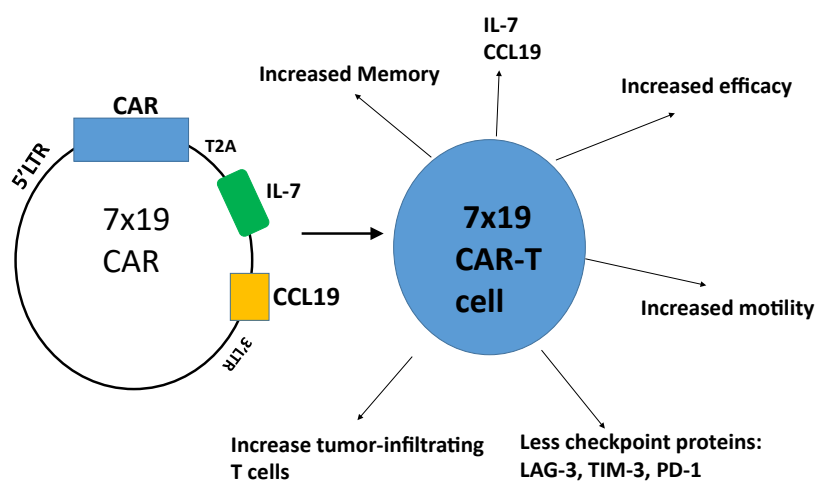

Figure 1. One of the steps of improving CAR-T therapy was proposed by using different cytokines during CAR-T cell expansion [6]. We proposed model of increasing CAR-T cell therapy approaches (Figure 2 [6])

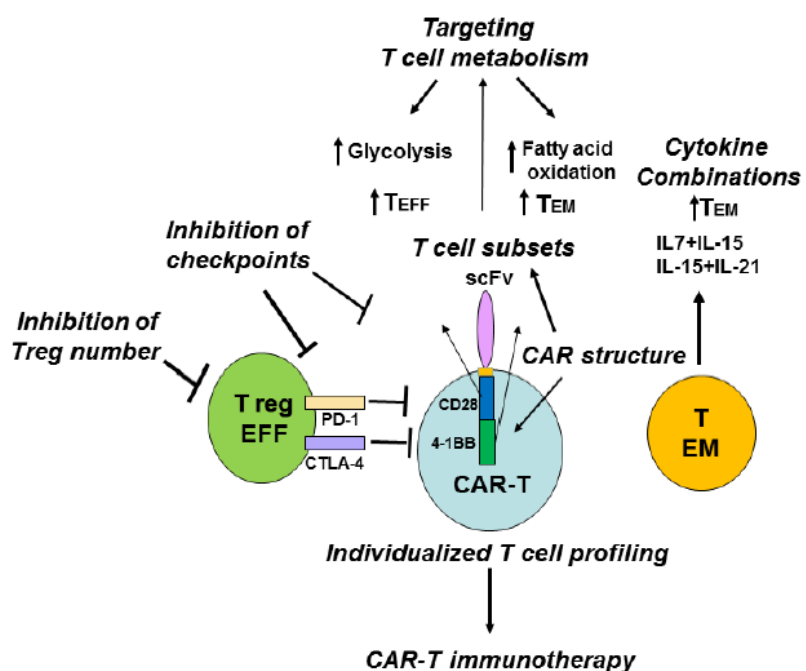

Figure 2. Different approaches to increase efficacy of CAR-T cell therapy. The figure is from [6]

*Correspondence to: Vita Golubovskaya, Probam Biotechnologies, 2600 Hilltop Drive, Richmond, CA, USA, E-mail: vita.gol@promab.com

Received: April 10, 2019; Accepted: April 17, 2019; Published: April 22, 2019 


\section{CD19-tag-CAR-T cells}

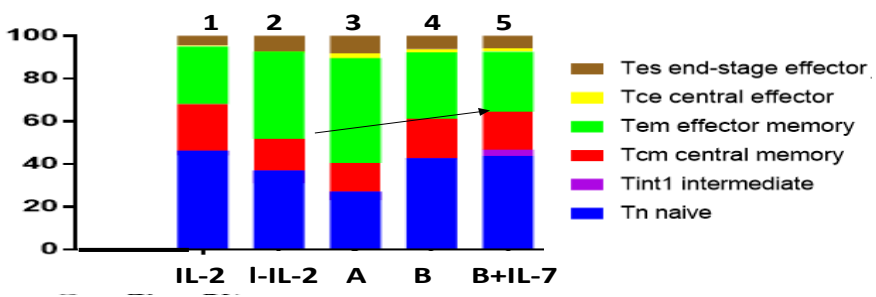

Figure 3. Different factors were used to increase percent of naïve and central memory cells resulting in CAR-T cells with less differentiated phenotype and increased memory of CAR-T cells. 1. CAR-T cells expanded 1 with high level of IL-2; 2 -low level IL-2; 3 low level plus A (AKT $1 / 2$ inhibitor) (marked A), 4. Low level IL-2 plus B (IL-15) (marked B); 5, low level IL-2 plus IL15+IL-7. The phenotype of CAR-T cells was detected by FACS with specific markers

generation CARs to overcome resistance of solid tumors with inhibitory microenvironment. The CAR designs with co-expressed growth factors or cytokines or other important proteins will be next step of improving efficacy of therapy in solid tumors.

\section{References}

1. Maus MV, June CH (2014) CARTs on the road for myeloma. Clin Cancer Res 20 3899-3901. [Crossref]

2. Porter DL, Hwang WT, Frey NV, Lacey SF, Shaw PA, et al. (2015). Chimeric antigen receptor $\mathrm{T}$ cells persist and induce sustained remissions in relapsed refractory chronic lymphocytic leukemia. Sci Transl Med 7: 303ra139.

3. Eshhar Z, Waks T, Gross G (2014) The emergence of T-bodies/CAR T cells. Cancer J 20: 123-126. [Crossref]

4. Kakarla S, Gottschalk S (2014) CAR T cells for solid tumors: armed and ready to go? Cancer J 20: 151-155. [Crossref]

5. Keishi Adachi, Yosuke Kano, Tomohiko Nagai, Namiko Okuyama, Yukimi Sakoda (2018) IL-7 and CCL19 expression in CAR-T cells improves immune cell infiltration and CAR-T cell survival in the tumor. Nat Biotechnol 36: 346-351.

6. Golubovskaya V, Wu L (2016) Different Subsets of T Cells, Memory, Effector Functions, and CAR-T Immunotherapy. Cancers 15: 3.

Copyright: $(2019$ Golubovskaya V. This is an open-access article distributed under the terms of the Creative Commons Attribution License, which permits unrestricted use, distribution, and reproduction in any medium, provided the original author and source are credited. 